\title{
POLYHEDRAL REALIZATION OF A THURSTON COMPACTIFICATION
}

\section{MATTHIEU GENDULPHE AND YOHEI KOMORI}

\begin{tabular}{|c|l|}
\hline Citation & OCAMI Preprint Series \\
\hline Issue Date & 2010 \\
\hline Type & Preprint \\
\hline Textversion & Author \\
\hline \multirow{2}{*}{ Relation } & $\begin{array}{l}\text { The following article has been submitted to Annales de la faculte des sciences de } \\
\text { Toulouse Mathematiques. } \\
\text { This is not the published version. Please cite only the published version. The } \\
\text { article has been published in final form at https://doi.org/10.5802/afst.1398 }\end{array}$ \\
\hline Is version of & https://doi.org/10.5802/afst.1398. \\
\hline
\end{tabular}

From: Osaka City University Advanced Mathematical Institute http://www.sci.osaka-cu.ac.jp/OCAMI/publication/preprint/preprint.html 


\title{
POLYHEDRAL REALIZATION OF A THURSTON COMPACTIFICATION
}

\author{
MATTHIEU GENDULPHE AND YOHEI KOMORI
}

\begin{abstract}
Let $\Sigma_{3}^{-}$be the connected sum of three real projective planes. We realize the Thurston compactification of the Teichmüller space Teich $\left(\Sigma_{3}^{-}\right)$as a simplex in $\mathbf{P}\left(\mathbf{R}^{4}\right)$. First, we define a map $L_{2}$ from Teich $\left(\Sigma_{3}^{-}\right)$into $\mathbf{P}\left(\mathbf{R}^{4}\right)$ in terms of some geodesic-length functions. We then introduce the similar triangle flow on Teich $\left(\Sigma_{3}^{-}\right)$to control the ratios between these lengths, and show that $L_{2}$ is an embedding. Finally, we study the natural extension of $L_{2}$ to the Thurston boundary using a triangulation of the projective space of measured foliations.
\end{abstract}

\section{INTRODUCTION}

In order to classify diffeomorphisms on a given compact surface $\Sigma$, W. P. Thurston built a compactification of the Teichmüller space Teich $(\Sigma)$ consisting in a closed ball lying in an infinite dimensional projective space. This Thurston compactification is simply defined as the closure of the image of the geodesic-length functions embedding

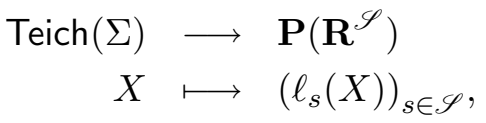

where $\mathscr{S}$ denotes the set of isotopy classes of nontrivial simple closed curves on $\Sigma$. Although the Thurston compactification is not a polytope, its boundary carries a piecewise integral projective structure, which interest stands in the identification of the Thurston boundary with the projective space of measured foliations. One would simplify the complicated combinatorial structure of the Thuston boundary and, in the same time, preserve its piecewise integral projective structure. In this direction, we address the problem of realizing the Thurston compactification as a finite convex integral polytope of a projective space of dimension $\operatorname{dim} T e i c h(\Sigma)$.

The first idea is to look at projections $\pi_{F}: \mathbf{P}\left(\mathbf{R}^{\mathscr{S}}\right) \rightarrow \mathbf{P}\left(\mathbf{R}^{F}\right)$ where $F$ is a subset of $\mathscr{S}$ of cardinal dimTeich $(\Sigma)+1$. It is in general very hard to decide whether such a projection defines an embedding of the Thurston compactification. Nevertheless, we know that for some $F$ the projection $\pi_{F}$ gives an embedding of the interior of the Thurston compactification (P. Schmutz [Sch93, Sch92]), and for some other $F$ an embedding of the Thurston boundary (U. Hamenstädt [Ham03]).

In this note we study the particular case of $\Sigma_{3}^{-}$, the connected sum of three real projective planes. Its Teichmüller space Teich $\left(\Sigma_{3}^{-}\right)$is of dimension three and quite easy to handle. We present an explicit embedding of its Thurston compactification into $\mathbf{P}\left(\mathbf{R}^{4}\right)$, and describe its image. More precisely, if $\alpha, \beta, \gamma, \alpha^{\prime}, \beta^{\prime}, \gamma^{\prime}$ in $\mathscr{S}$ satisfy a precise topological configuration which will be defined in $\S 3$, then

The first author was fully supported by the Fonds National Suisse de la Recherche Scientifique (SNF projects no. 200020-121506/1 and no. 200021-131967/1). The second author was partially supported by Grant-in-Aid for Scientific Research (19540194), Ministry of Education, Science and Culture of Japan. 
Theorem. The restriction of the following map induces an embedding of the Thurston compactification of Teich $\left(\Sigma_{3}^{-}\right)$into $\mathbf{P}\left(\mathbf{R}^{4}\right)$ which image is a projective simplex,

$$
\begin{aligned}
L_{2}: \mathbf{P}\left(\mathbf{R}^{\mathscr{S}}\right) & \longrightarrow \mathbf{P}\left(\mathbf{R}^{4}\right) \\
\left(x_{s}\right)_{s \in \mathscr{S}} & \longmapsto\left(x_{\alpha}: x_{\beta}: x_{\gamma}: x_{\alpha^{\prime}}+x_{\beta^{\prime}}+x_{\gamma^{\prime}}\right) .
\end{aligned}
$$

As we obtain the simplest polytope, this result is in some sense optimal. To prove that $L_{2}$ is projectively injective on the Teichmüller space, we introduce the similar triangle flow on Teich $\left(\Sigma_{3}^{-}\right)$. A trajectory of this flow preserve ratios between the length functions $\ell_{\alpha}, \ell_{\beta}$ and $\ell_{\gamma}$. Accurate estimates on the derivative of $\ell_{\alpha^{\prime}}, \ell_{\beta^{\prime}}$ and $\ell_{\gamma^{\prime}}$ along these trajectories enable us to conclude.

We also consider a projection $L_{1}:\left(x_{s}\right)_{s \in \mathscr{S}} \mapsto\left(x_{\alpha}: x_{\beta}: x_{\gamma}: x_{\sigma}\right)$ which restriction does not define an embedding of the Thurston compactification of Teich $\left(\Sigma_{3}^{-}\right)$. We show that in fact $L_{1}$ gives an embedding of another compactification, which is an interesting mixture of the Thurston compactification with the Teichmüller space of hyperbolic structures on $\Sigma_{3}^{-}$pinched at $\sigma(\S 6)$.

The text is organized as follows: in $\S 2$ we recall some basic facts about Teichmüller spaces and their Thurston compactification, in $\S 3$ we describe the topology and geometry of $\Sigma_{3}^{-}$, in $\S 4$ we introduce the similar triangle flow and study the behaviour of some length functions along its trajectories, and finaly in $\S 6$ we interpret the projective polyhedra obtained from $L_{1}$ and $L_{2}$.

Acknowledgement. Both authors are grateful to Prof. Ruth Kellerhals who makes possible this collaboration with the support of SNF projects no. 200020-121506/1 and no. 200021-131967/1. The second author wishes to thank her for her hospitality during his stay at the university of Fribourg.

\section{Preliminaries}

The reader can look at the classical references [Thu88] or to [FLP91] for more details on Teichmüller spaces and their Thuston compactification.

Let $\Sigma$ be a compact surface of negative Euler-Poincaré characteristic $\chi(\Sigma)<0$. A simple closed curve on $\Sigma$ is indivisible if it does not bound a disk or a Mobius strip. We will denote $\mathscr{S}$ the set of isotopy classes of indivisible simple closed curves on $\Sigma$, which do not retract into a boundary component.

2.1. Thurston compactification. The Teichmüller space Teich $(\Sigma)$ is the space of isotopy classes of hyperbolic metrics on $\Sigma$. When $\Sigma$ has nonempty boundary, we assume that the lengths of the boundary components are fixed. Endowed with its natural smooth structure, the Teichmüller space embeds into the projective space $\mathbf{P}\left(\mathbf{R}^{\mathscr{S}}\right)$ via length functions of geodesics. Its image is an open ball of dimension $\operatorname{dim}$ Teich $(\Sigma)=-3 \chi(\Sigma)-n$, where $n$ denotes the number of boundary components.

The Thurston boundary of the Teichmüller space is the boundary of its image in $\mathbf{P}\left(\mathbf{R}^{\mathscr{S}}\right)$. It is a topological sphere of dimension $\operatorname{dim} \operatorname{Teich}(\Sigma)-1$ denoted $\partial \operatorname{Teich}(\Sigma)$. Intersection functions indentify the Thurston boundary with the projectivised space of measured foliations $\operatorname{PMF}(\Sigma)$, and also with the projectivised space of measured geodesic laminations $\operatorname{PML}(\Sigma)$. The Thurston compactification of the Teichmüller space is the closure of its image in $\mathbf{P}\left(\mathbf{R}^{\mathscr{S}}\right)$, which turns out to be a closed ball. 
2.2. Integral points in the Thurston boundary. A curve system of $\Sigma$ is an isotopy class of finite unions of disjoint two-sided simple closed curves on $\Sigma$, none of which retracts into a point or a boundary component. We denote $\operatorname{CS}(\Sigma)$ the set of curve systems. The Thurston boundary possesses a piecewise integral projective structure. The set of integral points correspond exactly to $\operatorname{CS}(\Sigma)$ and is dense in $\partial \operatorname{Teich}(\Sigma)$.

Although $\partial \operatorname{Teich}(\Sigma)$ has no canonical simplicial decomposition, the curve complex $\mathrm{C}(\Sigma)$ is a simplicial complex which embedds canonically into $\partial \mathrm{Teich}(\Sigma)$. The set of vertices of $\mathrm{C}(\Sigma)$ is $\mathscr{S}$, and a collection $\left\{c_{1}, \ldots, c_{n}\right\} \subset \mathscr{S}$ defines a $(n-1)$-simplex if these curves have zero intersection numbers.

2.3. Notations and conventions. We denote $\Sigma_{g, n}\left(\operatorname{resp} . \Sigma_{g, n}^{-}\right)$the orientable (resp. the non-orientable) compact surface of genus $g$ with $n$ boundary components. A hyperbolic metric is a complete metric of constant sectional curvature -1 , with totally geodesic boundary. Unless otherwise is stated, geodesic means an indivisible simple closed geodesic which is not a boundary component. With this convention, the set of geodesics is in bijection with $\mathscr{S}$.

Instead of two-sided (resp. one-sided), we prefer to say that a simple closed curve is orientable (resp. non-orientable) if it is transversely orientable (resp. if it is not transversely orientable). Two isotopic non-orientable curves always intersect, but their intersection number as measured foliations is zero, so i $(c, c)=0$ for any element $c$ of $\mathscr{S}$. This makes the intersection number multiplicative.

If we need to explicit the lengths $b_{1}, \ldots, b_{n}$ of the boundary components, then we will use the following notation: $\operatorname{Teich}_{b_{1}, \ldots, b_{n}}\left(\Sigma_{g, n}^{ \pm}\right)$.

\section{Geometry and topology of the nON-ORIEntable surface of Genus 3}

In this part $X$ is a hyperbolic connected sum of three projective planes. In the first paragraphs, we classify geodesics of $X$ and look at a classical configuration which gives a nice affine embedding of Teich $\left(\Sigma_{3}^{-}\right)$. The last paragraphs are devoted to curves systems and projective measured foliations of $\Sigma_{3}^{-}$.

3.1. Simple closed geodesics. Results here are extracted from the article [Gen] of the first author. We have recently discovered that the proposition below was first obtained by M. Scharlemann in [Sch82].

Proposition 3.1. There is a unique simple closed geodesic $\sigma$ in $X$ which produces a one holed torus $\mathbb{T}_{X}$ after cutting.

This induces a canonical bijection between Teich $\left(\Sigma_{3}^{-}\right)$and $\cup_{b \in \mathbf{R}_{+}^{*}} \operatorname{Teich}_{b}\left(\Sigma_{1,1}\right)$.

Proposition 3.2. Let $\gamma$ be a simple closed geodesic of $X$ distinct from $\sigma$.

(1) If $\gamma$ is orientable, then $\gamma$ is disjoint from $\sigma$.

(2) If $\gamma$ is non-orientable, then $\gamma$ intersects $\sigma$ in exactly one point.

(3) There exists a unique simple closed geodesic $\gamma^{\prime} \neq \sigma$ disjoint from $\gamma$ which has opposite orientability. We say that $\gamma$ and $\gamma^{\prime}$ are duals.

The duality defines an involution of $\mathscr{S}$ which has $\sigma$ as a unique fixed point. The lengths of an orientable simple closed geodesic $\gamma$ and its dual $\gamma^{\prime}$ are related by

$$
\cosh \frac{\ell(\gamma)}{2}=\sinh \frac{\ell\left(\gamma^{\prime}\right)}{2} \sinh \frac{\ell(\sigma)}{2}
$$


The corresponding identity on the intersection numbers is

$$
\mathrm{i}(\gamma, \cdot)=\mathrm{i}\left(\gamma^{\prime}, \cdot\right)+\mathrm{i}(\sigma, \cdot) \text { on } \mathscr{S} \backslash\left\{\gamma^{\prime}, \sigma\right\} .
$$

These identities appear clearly on the figure 1 .
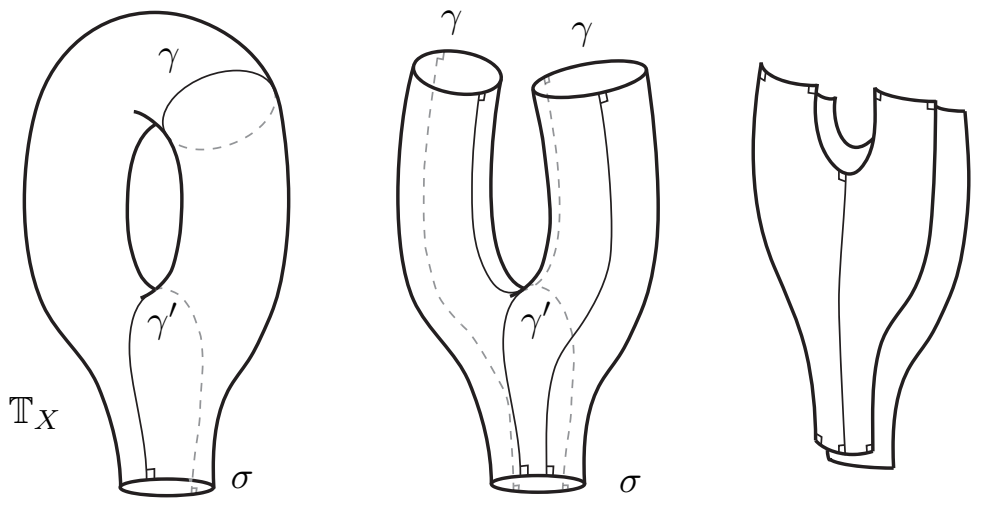

Figure 1. A geodesic and its dual

\subsection{Triangle embedding.}

Definition 3.1. A triangle is a triple $(\alpha, \beta, \gamma)$ of orientable simple closed geodesics with all intersection numbers equal to one.

Remark 3.1. A triple $(\alpha, \beta, \gamma)$ is a triangle if and only if its dual triple $\left(\alpha^{\prime}, \beta^{\prime}, \gamma^{\prime}\right)$ consists of three disjoint simple closed geodesics. The complement in $\Sigma_{3}^{-}$of these dual curves is a pair of pants.

Any triangle satisfies the geometric inequality (obvious after cutting the geodesics)

$$
\ell(\alpha)+\ell(\beta)+\ell(\gamma)>2 \ell(\sigma),
$$

and also the following identity

$$
\cosh ^{2} \frac{\ell(\sigma)}{2}=\left[\cosh \frac{\ell(\alpha)+\ell(\beta)}{2}-\cosh \frac{\ell(\gamma)}{2}\right]\left[\cosh \frac{\ell(\gamma)}{2}-\cosh \frac{\ell(\alpha)-\ell(\beta)}{2}\right](4)
$$

which comes from hyperbolic trigonometry in a right-angled hexagon.

Proposition 3.3. Let $(\alpha, \beta, \gamma)$ be a triangle, then the following map is an embedding

$$
\begin{aligned}
L: \operatorname{Teich}\left(\Sigma_{3}^{-}\right) & \longrightarrow \mathbf{R}^{3} \\
X & \longmapsto\left(\ell_{\alpha}(X), \ell_{\beta}(X), \ell_{\gamma}(X)\right)
\end{aligned}
$$

and its image is the following unbounded domain

$$
\begin{aligned}
\Delta=\left\{(a, b, c) \in \mathbf{R}^{3} \quad \mid\right. & b+c>a, c+a>b, a+b>c \text { and } \\
& {\left.\left[\cosh \frac{a+b}{2}-\cosh \frac{c}{2}\right]\left[\cosh \frac{c}{2}-\cosh \frac{a-b}{2}\right]>1\right\} . }
\end{aligned}
$$

It is well known that $L$ is smooth and injective (see [Sch92] for instance), one can easily show that $L$ is in fact a differential embedding with image $\Delta$.

Remarks 3.1. - Triangle inequalities imply that $a, b$ and $c$ are positive.

- The last inequality can be replaced by any symmetric one in $a, b, c$.

- $\Delta$ is invariant under multiplication by a scalar $t \geq 1$. 
3.3. Curve systems. Let $\lambda$ be a curve system. For every $c \in \mathscr{S}$ we will denote $\lambda_{c}$ the number of components of $\lambda$ isotopic to $c$ if $c$ is orientable, or to $c^{2}$ if $c$ is non-orientable.

Proposition 3.4. Let $\left(\alpha^{\prime}, \beta^{\prime}, \gamma^{\prime}\right)$ be a dual triple of a triangle. Then the following map is a bijection

$$
\begin{aligned}
\mathrm{CS}\left(\Sigma_{3}^{-}\right) & \longrightarrow \mathbf{Z}^{3} \\
\lambda & \longmapsto\left(\mathrm{i}\left(\alpha^{\prime}, \lambda\right)-\lambda_{\alpha^{\prime}}, \mathrm{i}\left(\beta^{\prime}, \lambda\right)-\lambda_{\beta^{\prime}}, \mathrm{i}\left(\gamma^{\prime}, \lambda\right)-\lambda_{\gamma^{\prime}}\right) .
\end{aligned}
$$

Proof. Let us consider a triple $\left(z_{\alpha}, z_{\beta}, z_{\gamma}\right) \in \mathbf{Z}^{3}$. We assume that each coordinate is nonnegative, but our argument also works for other cases. The triple $\left(2 z_{\alpha}, 2 z_{\beta}, 2 z_{\gamma}\right)$ is realized as Dehn-Thurston coordinates of some arc system $\bar{\lambda}$ of $\Sigma_{0,3}$ (see [LS04] for instance). As the intersection number of $\bar{\lambda}$ with each boundary is even, this arc system of $\Sigma_{0,3}$ induces a curve system $\lambda$ of $\Sigma_{3}^{-}$after antipodal identification of the boundaries. Using classical surface topology, we see that the intersection numbers of $\lambda$ with the curves coming from the boundaries are $z_{\alpha}, z_{\beta}$ and $z_{\gamma}$. This proves the sujectivity.

Let $\lambda, \kappa$ be two curve systems of $\Sigma_{3}^{-}$with same image through the above map. To simplify we assume that they have no component isotopic to $\alpha^{\prime}, \beta^{\prime}$ or $\gamma^{\prime}$. We look at $\lambda$ and $\kappa$ as integer measured geodesic laminations of $X$. Let $\bar{\lambda}$ and $\bar{\kappa}$ be their traces on the pair of pants $P_{X}$ obtained after cutting $\alpha^{\prime}, \beta^{\prime}$ and $\gamma^{\prime}$. Clearly $\bar{\lambda}$ and $\bar{\kappa}$ determine the same arc system on $P_{X}$ (see [LS04]). So, for each boundary of $P_{X}$, there is a canonical bijection between the points of $\lambda$ and $\kappa$ on this boundary. This bijection can be easily realized by an isotopy of the boundary which commutes with the antipodal map. These isotopies of the boundaries induce isotopies on $P_{X}$ and $X$ between $\lambda$ and $\kappa$ through geodesic arcs, proving the injectivity.

Remark 3.2. There are analogous bijections $\operatorname{CS}\left(\Sigma_{2,1}^{-}\right) \rightarrow \mathbf{Z}^{2}$ and $\operatorname{CS}\left(\Sigma_{1,2}^{-}\right) \rightarrow \mathbf{Z}$.

3.4. Scharlemann's description of $\operatorname{PMF}\left(\Sigma_{3}^{-}\right)$. M. Scharlemann gave in [Sch82] a nice description of the embedding $\mathrm{C}\left(\Sigma_{3}^{-}\right) \subset \operatorname{PMF}\left(\Sigma_{3}^{-}\right)$, based on the classical relation between $\mathrm{C}\left(\Sigma_{1,1}\right)$ and the Farey tesselation. Let us recall that, after a choice of a symplectic basis of the homology, simple closed geodesics in the one-holed torus correspond bijectively to pair $(p, q)$ of relatively prime integers, and so to rational numbers $p / q$ in $\mathbf{R} \cup\{\infty\}$. The intersection number between two pairs $(p, q)$ and $(r, s)$ is equal to the absolute value $|p s-r q|$ of the intersection form. Thus three geodesics correspond to the vertices of a triangle of the Farey tesselation if and only if they form a triangle in the one-holed torus.

The boundary $\partial \operatorname{Teich}\left(\Sigma_{1,1}\right)$ embeds piecewise linearly and canonically into a circle in $\partial \operatorname{Teich}\left(\Sigma_{3}^{-}\right) \simeq \mathbb{S}^{2}$, and so divides it into two hemispheres. One of these hemispheres contains only one vertex of $\mathrm{C}\left(\Sigma_{3}^{-}\right)$in its interior, it corresponds to $\sigma$ and is related to all the vertices in $\partial \operatorname{Teich}\left(\Sigma_{1,1}\right)$. The other contains all the vertices corresponding to dual curves of orientable curves in his interior, the configuration of the curve complex in this hemisphere is a slightly modified version of the Farey tesselation: let us look at the hemisphere as the unit disk, if $z(p, q)$ represents the orientable geodesic with coordiantes $(p, q)$, then the point $z^{\prime}(p, q)=z(p, q) /(1+1 / q)$ represents its dual geodesic. The segment $\left[z(p, q), z^{\prime}(p, q)\right]$ is an edge of the curve complex, the other edges are between points $z^{\prime}(p, q)$ and $z^{\prime}(r, s)$ with $|p s-r q|=1$. See figure 2 for a picture where $(\alpha, \beta, \gamma)$ is a triangle. 
In fact, for any compact non-orientable surface $\Sigma$, points corresponding to nonorientable geodesics are isolated in $\operatorname{PMF}(\Sigma)$, but their closure contains all geodesics. This was proved by M. Scharlemann in [Sch82].

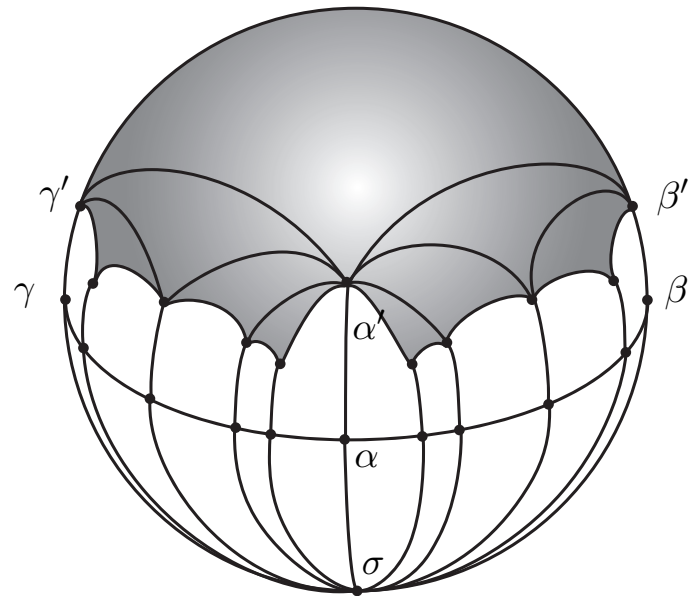

FiguRE 2. Scharlemann's picture

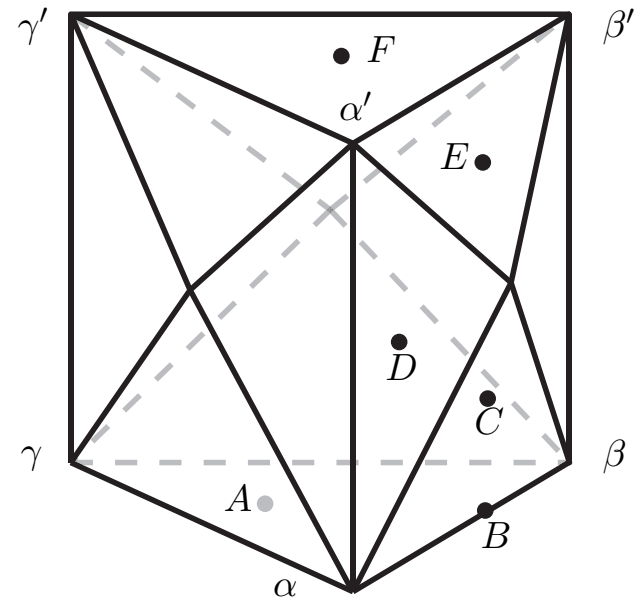

FIGURE 3

3.5. A simplicial description of $\operatorname{PMF}\left(\Sigma_{3}^{-}\right)$. Up to isotopy and multiplication by a positive constant, there are only two measured foliations on the Mobius band: foliations with closed leaves, and foliations with leaves transverse to the boundary. Let $\alpha^{\prime}, \beta^{\prime}$ and $\gamma^{\prime}$ be three disjoint indivisible simple closed curves which bound a pair of pants in $\Sigma_{3}^{-}$. Any measured foliation on $\Sigma_{3}^{-}$is isotopic to a measured foliation such that each of these curves is a leave, a cycle of leaves, or is transverse to the leaves. If one of these curves is a leave, then it admits a maximal Mœbius neighborhood foliated by closed leaves.

The complement of these curves and their maximal Mœbius neighborhoods is a pair of pants $P$, or eventually a graph if the support of the measure is contained in the maximal Mœbius neighborhoods. In the first case, the measured foliation induced on $P$ is good, i.e. none of the boundaries is a leave. Then, using the classification of good foliations on $P$ given in [FLP91] exposé $6 \S$ II, we classify measured foliations on $\Sigma_{3}^{-}$(figure 4 ). The measures of the maximal Mobius neighborhoods can change the dimension of simplices, for instance compare simplices (4), (5) and (6) in figure 4 of [FLP91] exposé 6 with their corresponding simplices in figure 4 . In the case where $P$ is graph, the measured foliation is isotopic to the foliation $F$ of the figure 4, it gives a new 2-simplex. We have described a simplicial decomposition of $\operatorname{PMF}\left(\Sigma_{3}^{-}\right)$, we draw a global picture of it in figure 3 .

\section{Monotonicity of LEngth FUnCtions Under the Similar triangle Flow}

In $\S 5$ we will use the similar triangle flow to show the injectivity of some map from Teich $\left(\Sigma_{3}^{-}\right)$into $\mathbf{P}\left(\mathbf{R}^{4}\right)$. Two points of Teich $\left(\Sigma_{3}^{-}\right)$with same image with respect to these maps belong to the same trajectory of the similar triangle flow. So, we have to study the behaviour of certain length functions along these trajectories to prove that distinct points have distinct images. 


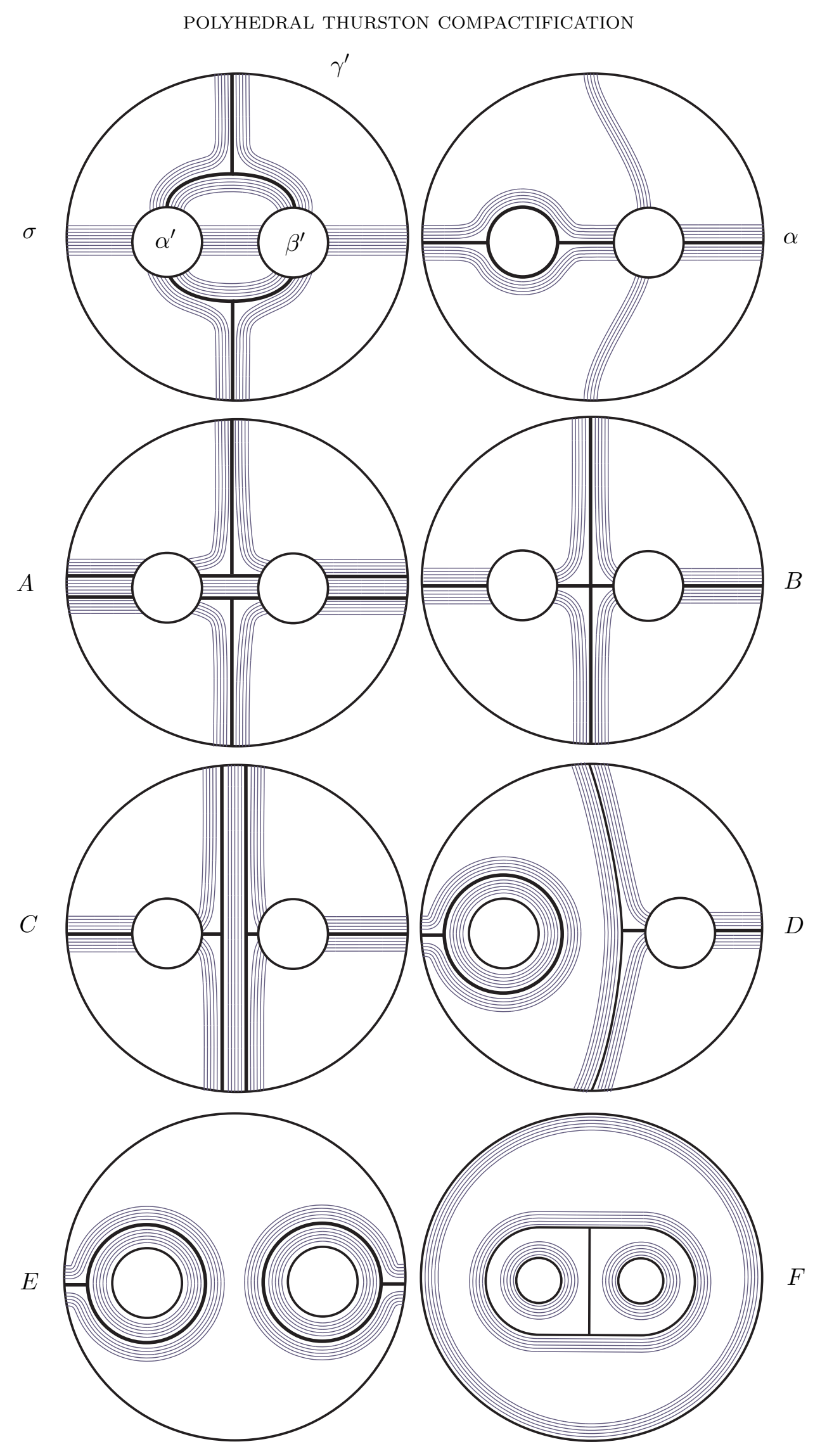

Figure 4 
4.1. Similar triangle flow. Let us consider the 1-parameter subgroup $\left\{x \mapsto e^{s} x\right\}_{s \in \mathbf{R}}$ coming from the radial linear vector field associated to the identity map of $\mathbf{R}^{3}$. Its composition with the logarithm defines a continuous multiplicative homomorphism

$$
\begin{array}{ccccc}
\phi \quad \mathbf{R}_{+}^{*} & \longrightarrow \mathbf{R} & \longrightarrow \operatorname{Diff}\left(\mathbf{R}^{3}\right) \\
t & \longmapsto & \ln (t) & \longmapsto & \phi_{t}: x \mapsto t x
\end{array} .
$$

Let $x=(a, b, c)$ be a point in the positive cone. It follows from remark 3.1 that the trace of the trajectory $\phi_{t}(x)$ on $\Delta$ is the half-line $\left\{t x ; t>t_{x}\right\}$ where $t_{x}$ is the unique positive solution of

$$
\left(\cosh \frac{a+b}{2} t-\cosh \frac{c}{2} t\right)\left(\cosh \frac{c}{2} t-\cosh \frac{a-b}{2} t\right)=1 .
$$

Let us fix a triangle $(\alpha, \beta, \gamma)$.

Definition 4.1. We call similar triangle flow with respect to $(\alpha, \beta, \gamma)$ the pullback of $\phi$ by $L$. We denote it $\triangle(X, t)$, or simply $\triangle(t)$ when $X$ is fixed.

The frontier of $\Delta$ can be canonically identified with the Teichmüller space of cusped tori, or equivalently with the Teichmüller space of hyperbolic structures on $\Sigma_{3}^{-}$noded at $\sigma$. The triangle flow can be extended to this space.

We now fix a point $X$ in Teich $\left(\Sigma_{3}^{-}\right)$. The trajectory $\triangle(t)$ is defined on an open interval $] t_{X},+\infty\left[\subset \mathbf{R}_{+}^{*}\right.$ containing 1 . The geometrical meaning of the similar triangle flow can be expressed as follows: for any $Y \in \operatorname{Teich}\left(\Sigma_{3}^{-}\right)$and any $\left.t \in\right] t_{X},+\infty[$

$$
\triangle(t)=Y \Longleftrightarrow\left\{\begin{array}{l}
\ell_{\alpha}(Y)=t \ell_{\alpha}(X) \\
\ell_{\beta}(Y)=t \ell_{\beta}(X) \\
\ell_{\gamma}(Y)=t \ell_{\gamma}(X)
\end{array} .\right.
$$

In the sequel we will denote $\eta(t)$ the length of any geodesic $\eta$ at the point $\triangle(t)$.

4.2. Monotonicity of $\sigma(t)$.

Proposition 4.1. For any $t>t_{X}$ we have

$$
\frac{\mathrm{d} \sigma}{\mathrm{d} t}(t)>\frac{\alpha(1)+\beta(1)+\gamma(1)}{2} .
$$

Proof. For simplicity we pose $a=\alpha(1), b=\beta(1)$ and $c=\gamma(1)$. In view of (4)

$$
\sigma(t)=2 \cosh ^{-1} \sqrt{f(t)}
$$

with

$$
f(t)=\left(\cosh \frac{a+b}{2} t-\cosh \frac{c}{2} t\right)\left(\cosh \frac{c}{2} t-\cosh \frac{a-b}{2} t\right) .
$$

By the following simple estimation

$$
\frac{\mathrm{d}}{\mathrm{d} t} 2 \cosh ^{-1} \sqrt{f(t)}=\frac{f^{\prime}(t)}{\sqrt{f(t)} \sqrt{f(t)-1}}>\frac{f^{\prime}(t)}{f(t)},
$$

it suffices to show that

$$
\frac{f^{\prime}(t)}{f(t)}>\frac{a+b+c}{2}=\frac{\alpha(1)+\beta(1)+\gamma(1)}{2} .
$$

In practice

$$
\frac{f^{\prime}(t)}{f(t)}=\frac{\frac{\mathrm{d}}{\mathrm{d} t}\left(\cosh \frac{a+b}{2} t-\cosh \frac{c}{2} t\right)}{\cosh \frac{a+b}{2} t-\cosh \frac{c}{2} t}+\frac{\frac{\mathrm{d}}{\mathrm{d} t}\left(\cosh \frac{c}{2} t-\cosh \frac{a-b}{2} t\right)}{\cosh \frac{c}{2} t-\cosh \frac{a-b}{2} t} .
$$


But for any $q>p>0$ and any $t>0$

$$
\begin{aligned}
\frac{\mathrm{d}}{\mathrm{d} t}(\cosh q t-\cosh p t) & =q \sinh q t-p \sinh p t \\
& >q(\sinh q t-\sinh p t)=q \int_{p t}^{q t} \cosh (s) \mathrm{d} s \\
& >q(\cosh q t-\cosh p t)=q \int_{p t}^{q t} \sinh (s) \mathrm{d} s .
\end{aligned}
$$

Thus, from (5) we conclude that

$$
\frac{f^{\prime}(t)}{f(t)}>\frac{a+b}{2}+\frac{c}{2}=\frac{a+b+c}{2}
$$

4.3. Monotonicity of $\alpha^{\prime}(t), \beta^{\prime}(t)$ and $\gamma^{\prime}(t)$.

Proposition 4.2. For any $t>t_{X}$ we have

$$
\frac{\mathrm{d} \alpha^{\prime}}{\mathrm{d} t}(t), \frac{\mathrm{d} \beta^{\prime}}{\mathrm{d} t}(t), \frac{\mathrm{d} \gamma^{\prime}}{\mathrm{d} t}(t)<0
$$

Proof. Let us consider the case of $\alpha^{\prime}$. From (1) we have

$$
\sinh \left(\frac{\alpha^{\prime}(t)}{2}\right) \sinh \left(\frac{\sigma(t)}{2}\right)=\cosh \left(\frac{\alpha(t)}{2}\right)=\cosh \left(\frac{t \alpha(1)}{2}\right) \quad\left(t>t_{X}\right)
$$

taking the derivative with respect to $t$ we obtain

$$
\frac{\mathrm{d} \alpha^{\prime}}{\mathrm{d} t}(t) \cosh \left(\frac{\alpha^{\prime}(t)}{2}\right) \sinh \left(\frac{\sigma(t)}{2}\right)+\frac{\mathrm{d} \sigma}{\mathrm{d} t}(t) \sinh \left(\frac{a^{\prime}(t)}{2}\right) \cosh \left(\frac{\sigma(t)}{2}\right)=\alpha(1) \sinh \left(\frac{t \alpha(1)}{2}\right) .
$$

Because of proposition 4.1 and triangle inequality

$$
\frac{\mathrm{d} \sigma}{\mathrm{d} t}(t)>\frac{\alpha(1)+\beta(1)+\gamma(1)}{2}>\alpha(1) .
$$

Hence

$$
\begin{aligned}
\frac{\mathrm{d} a^{\prime}}{\mathrm{d} t}(t) \cosh \left(\frac{\alpha^{\prime}(t)}{2}\right) \sinh \left(\frac{\sigma(t)}{2}\right) & =\alpha(1) \sinh \left(\frac{t \alpha(1)}{2}\right)-\frac{\mathrm{d} \sigma}{\mathrm{d} t}(t) \sinh \left(\frac{\alpha^{\prime}(t)}{2}\right) \cosh \left(\frac{\sigma(t)}{2}\right) \\
& <\alpha(1) \sinh \left(\frac{t \alpha(1)}{2}\right)-\alpha(1) \sinh \left(\frac{\alpha^{\prime}(t)}{2}\right) \cosh \left(\frac{\sigma(t)}{2}\right) \\
& <\alpha(1) \sinh \left(\frac{t \alpha(1)}{2}\right)-\alpha(1) \sinh \left(\frac{\alpha^{\prime}(t)}{2}\right) \sinh \left(\frac{\sigma(t)}{2}\right) \\
& <\alpha(1) \sinh \left(\frac{t \alpha(1)}{2}\right)-\alpha(1) \cosh \left(\frac{t a}{2}\right) \\
& <0
\end{aligned}
$$

which implies $\frac{\mathrm{d} \alpha^{\prime}}{\mathrm{d} t}(t)<0$. Identical proofs work for $\beta^{\prime}$ and $\gamma^{\prime}$.

\section{Two polyhedral Realizations of Teich $\left(\Sigma_{3}^{-}\right)$in $\mathbf{P}\left(\mathbf{R}^{4}\right)$}

In this part we give two embeddings of Teich $\left(\Sigma_{3}^{-}\right)$into $\mathbf{P}\left(\mathbf{R}^{4}\right)$. The injectivity of these embeddings comes from the monotonicity of $\ell_{\sigma}, \ell_{\alpha^{\prime}}, \ell_{\beta^{\prime}}$ and $\ell_{\gamma^{\prime}}$ along the similar triangle flow. We fix a triangle $(\alpha, \beta, \gamma)$ for all this part. 


\subsection{Embeddings defined via length functions.}

Theorem 5.1. The following maps are embeddings of Teich $\left(\Sigma_{3}^{-}\right)$into $\mathbf{P}\left(\mathbf{R}^{4}\right)$ :

$$
\begin{aligned}
L_{1}: \operatorname{Teich}\left(\Sigma_{3}^{-}\right) & \longrightarrow \mathbf{P}\left(\mathbf{R}^{4}\right) \\
X & \longmapsto\left(\ell_{\alpha}(X): \ell_{\beta}(X): \ell_{\gamma}(X): \ell_{\sigma}(X)\right) \\
L_{2}: \operatorname{Teich}\left(\Sigma_{3}^{-}\right) & \longrightarrow \mathbf{P}\left(\mathbf{R}^{4}\right) \\
X & \longmapsto\left(\ell_{\alpha}(X): \ell_{\beta}(X): \ell_{\gamma}(X): \ell_{\alpha^{\prime}+\beta^{\prime}+\gamma^{\prime}}(X)\right)
\end{aligned}
$$

Proof. Let us prove the injectivity of $L_{1}$. The system of length functions $\left(\ell_{\alpha}, \ell_{\beta}, \ell_{\gamma}\right)$ defines an injective map from Teich $\left(\Sigma_{3}^{-}\right)$into $\mathbf{R}^{3}$ by proposition 3.3. So, it suffices to show that two points $X, Y \in \operatorname{Teich}\left(\Sigma_{3}^{-}\right)$with same image $L_{1}(X)=L_{1}(Y)$ have same lengths with respect to $\alpha, \beta$ and $\gamma$.

These two points belong to the same trajectory under the similar triangle flow. Without loss of generality, we assume $Y=\triangle(t)$ for some $t \geq 1$. On one hand, by definition of the similar triangle flow, we have

$$
\ell_{\alpha}(Y)=\alpha(t)=t \alpha(1)=t \ell_{\alpha}(X)
$$

which implies by proportionality of lengths

$$
\sigma(t)=\ell_{\sigma}(Y)=t \ell_{\sigma}(X)=t \sigma(1)
$$

On another hand, from proposition 4.1 and (3) it comes that

$$
\begin{aligned}
\sigma(t) & =\sigma(1)+\int_{1}^{t} \frac{\mathrm{d} \sigma}{\mathrm{d} s}(s) \mathrm{d} s \\
& \geq \sigma(1)+(t-1) \frac{\alpha(1)+\beta(1)+\gamma(1)}{2} \\
& \geq \sigma(1)+(t-1) \sigma(1),
\end{aligned}
$$

with equality if and only if $t=1$. We conclude that $t=1$, thus $X$ and $Y$ have same lengths with respect to $\alpha, \beta, \gamma$ and $\sigma$.

The same proof also works for $L_{2}$. We just have to make few modifications: we consider $\left(\alpha^{\prime}+\beta^{\prime}+\gamma^{\prime}\right)(t)$ instead of $\sigma(t)$, and we use proposition 4.2 instead of proposition 4.1.

It remains to show that the $L_{i}$ are submersions, or equivalently that the maps $\left(\ell_{\alpha}, \ell_{\beta}, \ell_{\gamma}, \ell_{\sigma}\right)$ and $\left(\ell_{\alpha}, \ell_{\beta}, \ell_{\gamma}, \ell_{\alpha^{\prime}+\beta^{\prime}+\gamma^{\prime}}\right)$ are transverse to lines of $\mathbf{R}^{4}$ passing through the origin. Let us consider a germ of curve $c=\left(c_{\alpha}, c_{\beta}, c_{\gamma}, c_{\sigma}\right)$ in the image of $\left(\ell_{\alpha}, \ell_{\beta}, \ell_{\gamma}, \ell_{\sigma}\right)$, which is tangent to a line of $\mathbf{R}^{4}$ passing through the origin. Then $\left(c_{\alpha}, c_{\beta}, c_{\gamma}\right)$ is a germ of curve in $L\left(\right.$ Teich $\left.\left(\Sigma_{3}^{-}\right)\right)$tangent to a line of $\mathbf{R}^{3}$ passing through the origin, and so tangent to the image $L(\triangle(t))$ of a trajectory of the similar triangle flow. As $L$ is an embedding (proposition 3.3) it comes that $c$ is tangent to the image of $\triangle(t)$ by $\left(\ell_{\alpha}, \ell_{\beta}, \ell_{\gamma}, \ell_{\sigma}\right)$. This is not possible according to proposition 4.1 and inequality (3), so $L_{1}$ is a submersion. The same argument works also for $L_{2}$.

\subsection{Images as convex projective polyhedra.}

Corollary 5.2. The images of $L_{1}$ and $L_{2}$ are convex polyhedra in $\mathbf{P}\left(\mathbf{R}^{4}\right)$ : 
- the image $L_{1}\left(\operatorname{Teich}\left(\Sigma_{3}^{-}\right)\right)$is the convex projective polyhedron

$$
\begin{array}{r}
\Delta_{1}:=\left\{(a: b: c: d) \in \mathbf{P}\left(\mathbf{R}^{4}\right) \mid a+b>c, b+c>a, c+a>b \text { and } d>0\right. \\
\text { and } a+b+c>2 d\} .
\end{array}
$$

- the image $L_{2}\left(\operatorname{Teich}\left(\Sigma_{3}^{-}\right)\right)$is the simplex

$$
\Delta_{2}:=\left\{(a: b: c: d) \in \mathbf{P}\left(\mathbf{R}^{4}\right) \quad \mid \quad b+c>a, c+a>b, a+b>c \text { and } d>0\right\} .
$$

Remark 5.1. Triangle inequalities imply that $a, b$ and $c$ are nonnegative.

Proof. We clearly have $L_{2}\left(\operatorname{Teich}\left(\Sigma_{3}^{-}\right)\right) \subset \Delta_{2}$, and also $L_{1}\left(\operatorname{Teich}\left(\Sigma_{3}^{-}\right)\right) \subset \Delta_{1}$ by means of (3). So it remains to show that $\Delta_{i} \subset L_{i}\left(\operatorname{Teich}\left(\Sigma_{3}^{-}\right)\right)$for $i=1,2$.

Let us consider $(a, b, c, d) \in \mathbf{R}^{4}$ satisfying the conditions in the definition of $\Delta_{i}$. Up to multiplication by a positive scalar, we can also assume that $(a, b, c)=L_{i}(X)$ for some $X$ in $\operatorname{Teich}\left(\Sigma_{3}^{-}\right)$. We claim that there exists $\left.t \in\right] t_{X},+\infty[$ such that

$$
(\alpha: \beta: \gamma: \sigma)(t)=(a: b: c: d) .
$$

This will prove that $(a: b: c: d) \in L_{i}\left(\operatorname{Teich}\left(\Sigma_{3}^{-}\right)\right)$and conclude the proof.

Case of $L_{1}$. By definition of $t_{X}$ we have $\sigma(t) \rightarrow 0$ when $t \rightarrow t_{X}$, so

$$
d t>\sigma(t)
$$

for $t$ sufficiently close to $t_{X}$. The proposition 4.1 and $a+b+c>2 d$ leads to

$$
\begin{aligned}
2 \sigma(t) & \geq 2 \sigma(1)+(a+b+c)(t-1) \\
& \geq 2 \sigma(1)-2 d+(a+b+c-2 d)(t-1)+2 d t \\
& \geq 2 d t
\end{aligned}
$$

for $t$ sufficiently large. As a consequence, there is a $t_{0}>t_{x}$ such that $\sigma\left(t_{0}\right)=d t_{0}$, and $(\alpha, \beta, \gamma, \sigma)\left(t_{0}\right)=t_{0}(a, b, c, d)$ as we claimed.

Case of $L_{2}$. By the collar lemma

$$
\sigma(t) \underset{t \rightarrow t_{X}}{\longrightarrow} 0 \text { implies }\left(\alpha^{\prime}+\beta^{\prime}+\gamma^{\prime}\right)(t) \underset{t \rightarrow t_{X}}{\longrightarrow}+\infty .
$$

So $\left(\alpha^{\prime}+\beta^{\prime}+\gamma^{\prime}\right)(t)>d t$ for $t$ sufficiently close to $t_{X}$. But $\left(\alpha^{\prime}+\beta^{\prime}+\gamma^{\prime}\right)(t)$ is a decreasing function (proposition 4.2), thus $\left(\alpha^{\prime}+\beta^{\prime}+\gamma^{\prime}\right)(t)<d t$ for $t$ sufficiently large. As a byproduct there exists $t_{0}>t_{X}$ such that

$$
\left(\alpha, \beta, \gamma, \alpha^{\prime}+\beta^{\prime}+\gamma^{\prime}\right)\left(t_{0}\right)=t_{0}(a, b, c, d),
$$

as we claimed.

\section{INTERPRETATION OF THE BOUNDARIES OF $\Delta_{1}$ AND $\Delta_{2}$}

Let us fix a triangle $(\alpha, \beta, \gamma)$. There exists a unique curve $\eta \neq \alpha$ such that $(\eta, \beta, \gamma)$ is a triangle, we denote $\alpha^{\star}$ the dual curve of $\eta$. 


\begin{tabular}{|c|c|c|c|c|c|}
\hline point & coordinates & point & coordinates & point & coordinates \\
\hline \hline$A$ & $(0: 1: 1: 2)$ & $A^{\prime}$ & $(0: 1: 1: 0)$ & $A^{\star}$ & $(2: 1: 1: 1)$ \\
$B$ & $(1: 0: 1: 2)$ & $B^{\prime}$ & $(1: 0: 1: 0)$ & $B^{\star}$ & $(1: 2: 1: 1)$ \\
$C$ & $(1: 1: 0: 2)$ & $C^{\prime}$ & $(1: 1: 0: 0)$ & $C^{\star}$ & $(1: 1: 2: 1)$ \\
$E$ & $(0: 1: 1: 1)$ & $F$ & $(1: 0: 1: 1)$ & $G$ & $(1: 1: 0: 1)$ \\
$S$ & $(0: 0: 0: 1)$ & & & & \\
\hline
\end{tabular}

TABle 1. Some points in $\mathbf{P}\left(\mathbf{R}^{4}\right)$ and their coordinates

6.1. Description of the boundaries. The closure of $\Delta_{2}$ is the simplex spanned by $A^{\prime}, B^{\prime}, C^{\prime}$ and $S$. Whereas the closure of $\Delta_{1}$ is the truncated simplex with vertices $A^{\prime}, B^{\prime}, C^{\prime}$, and $E, F, G$. All these points are defined in the table 6.1 below.

If these configurations are not obvious, one can use the projective transformation

$$
(a: b: c: d) \mapsto((b+c)-a:(a+c)-b:(a+b)-c: a+b+c+d)
$$

which sends respectively $\Delta_{2}$ and $\Delta_{1}$ on $\{(x: y: z: t) \mid x, y, z>0$ and $t>x+y+z\}$ and $\left\{(x: y: z: t) \mid x, y, z>0\right.$ and $\left.t>x+y+z>\frac{2}{3} t\right\}$. Then, everything becomes clear in the affine chart $\{t=1\}$.

6.2. Interpretation. Each embedding $L_{i}$ factors through $L$, and also through the canonical embedding of Teich $\left(\Sigma_{3}^{-}\right)$into $\mathbf{P}\left(\mathbf{R}^{\mathscr{S}}\right)$. The situation is represented in the commutative diagram below, where the maps $\phi_{i}$ and $\psi_{i}$ are quite obvious.

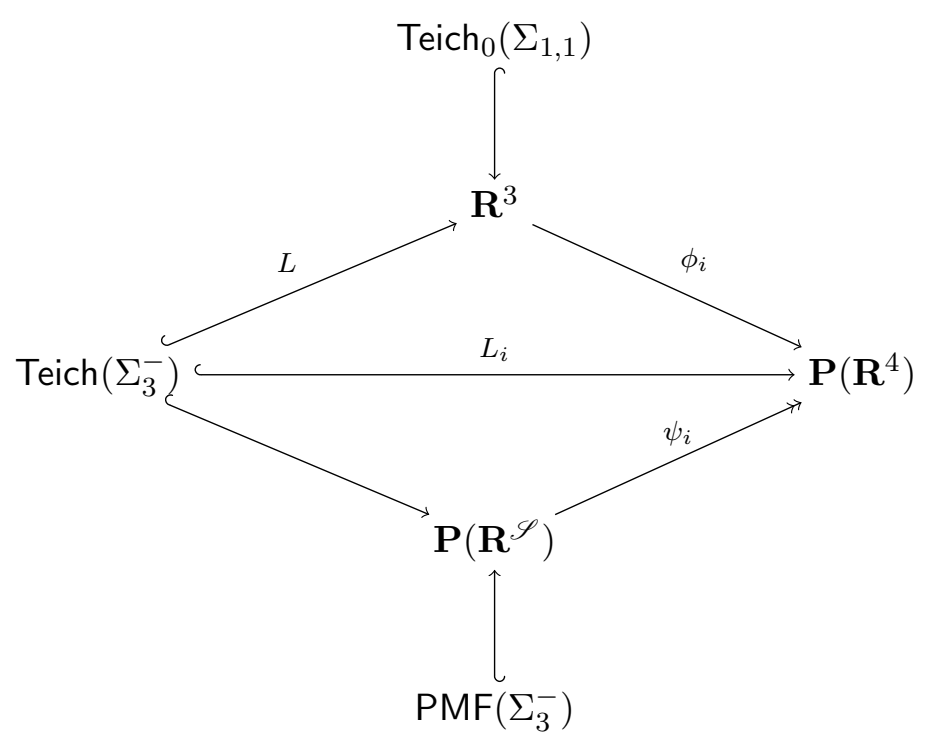

6.2.1. Case of $\Delta_{1}$. The map $\phi_{1}$ extends differentiably to the closure of $\Delta$, and induces an embedding of the Teichmüller space of cusped tori into $\mathbf{P}\left(\mathbf{R}^{4}\right)$, which is simply $\left(\ell_{\alpha}: \ell_{\beta}: \ell_{\gamma}: 0\right)$. The image of this embedding is exactly the interior of the face $\left\langle A^{\prime}, B^{\prime}, C^{\prime}\right\rangle$ of $\Delta_{1}$.

The map $\psi_{1}$ extends coutinuously to the set of points in the Thurston boundary satisfying the condition $\left(\mathrm{i}_{\alpha}, \mathrm{i}_{\beta}, \mathrm{i}_{\gamma}, \mathrm{i}_{\sigma}\right) \neq 0$. This set turns out to be $\partial \operatorname{Teich}\left(\Sigma_{3}^{-}\right) \backslash\{\sigma\}$. The vertices of the triangulation of $\operatorname{PMF}\left(\Sigma_{3}^{-}\right) \simeq \partial \operatorname{Teich}\left(\Sigma_{3}^{-}\right)$studied in $\S 3.5$ are sent on vertices, and barycenters of faces, of $\Delta_{1}$ (figure 5 ). Thus, the extension 
of $\psi_{1}$ induces a piecewise integral projective isomorphism from the complement in $\operatorname{PMF}\left(\Sigma_{3}^{-}\right)$of the interior of the simplex $\langle\alpha, \beta, \gamma\rangle$, to the complement in $\partial \Delta_{1}$ of the interior of the simplex $\left\langle A^{\prime}, B^{\prime}, C^{\prime}\right\rangle$. The extension of $\psi_{1}$ mashes $\langle\alpha, \beta, \gamma\rangle \backslash\{\sigma\}$ onto the boundary of $\left\langle A^{\prime}, B^{\prime}, C^{\prime}\right\rangle$. More precisely, a point $X$ in $\langle\alpha, \beta, \gamma\rangle \backslash\{\sigma\}$ has same image as the point $Y$ on the boundary of the simplex such that $X$ belongs to $[\sigma, Y]$.

Interpretation. The polyhedon $\Delta_{1}$ is a compactification of the Teichmüller spaces $\cup_{b>0} \operatorname{Teich}_{b}\left(\Sigma_{1,1}\right)$. Its boundary decomposes into two pieces. One piece corresponds exactly to the set of projective measured foliations of the one-holed torus, where leaves transverse to the boundary are allowed. The other piece corresponds exactly to the Teichmüller space of cusped tori. The frontier between these two pieces is the Thurston boundary of $\operatorname{Teich}_{b}\left(\Sigma_{1,1}\right)$, which does not depend on the fixed length $b>0$.

We have to take care of the way we define measured foliations. If we work with $\operatorname{Teich}_{b}\left(\Sigma_{1,1}\right)$ ( $b \geq 0$ fixed) then we consider measured foliations as in [FLP91] exposé $11 \S 1$. But if we work on $\cup_{b>0} \operatorname{Teich}_{b}\left(\Sigma_{1,1}\right)$, then we allowed measured foliations to have leaves transverse to the boundary. Nevertheless, we still assume that no closed leave is isotopic to the boundary, that's why the simplex $\langle\alpha, \beta, \gamma\rangle$ collapses.

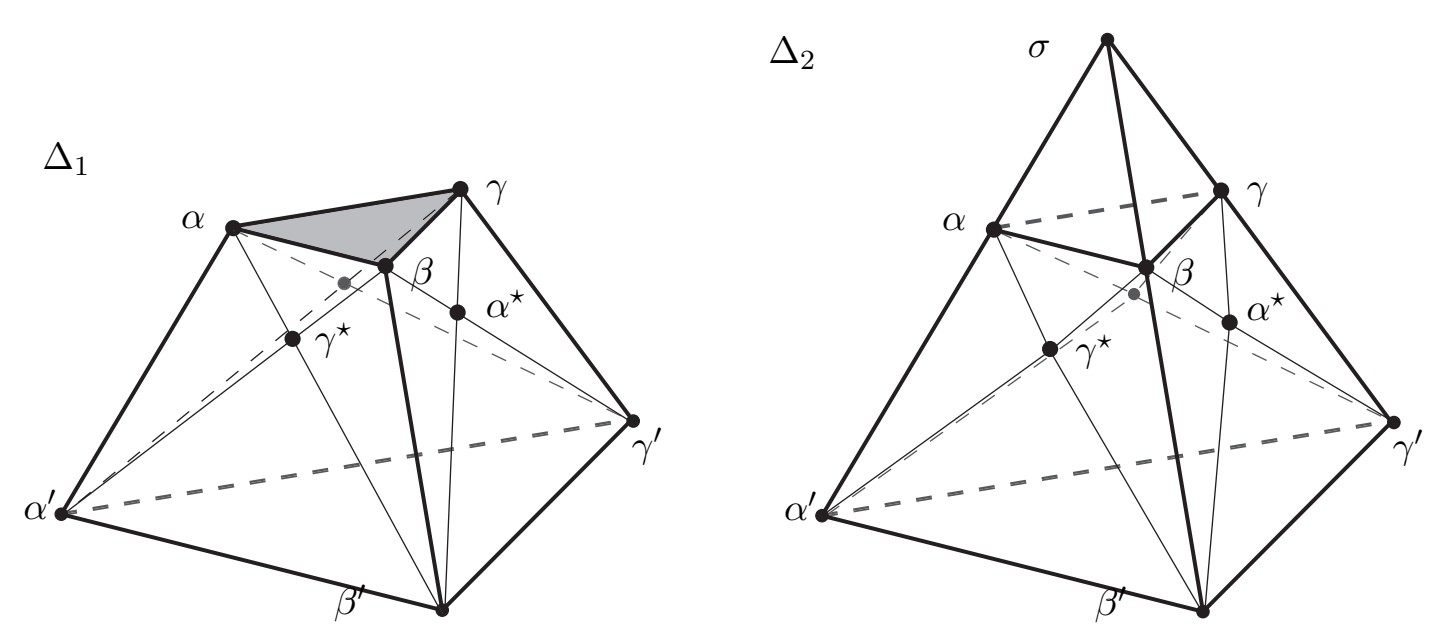

Figure 5. Polyhedral representations

\begin{tabular}{c|c|c|c|c|c|c|c|} 
& $\mathrm{i}_{\alpha}$ & $\mathrm{i}_{\beta}$ & $\mathrm{i}_{\gamma}$ & $\mathrm{i}_{\sigma}$ & $\mathrm{i}_{\alpha^{\prime}+\beta^{\prime}+\gamma^{\prime}}$ & in $\Delta_{1}$ & in $\Delta_{2}$ \\
\hline \hline$\alpha$ & 0 & 1 & 1 & 0 & 2 & $A^{\prime}$ & $A$ \\
$\beta$ & 1 & 0 & 1 & 0 & 2 & $B^{\prime}$ & $B$ \\
$\gamma$ & 1 & 1 & 0 & 0 & 2 & $C^{\prime}$ & $C$ \\
$\sigma$ & 0 & 0 & 0 & 0 & 3 & & $S$ \\
$\alpha^{\prime}$ & 0 & 1 & 1 & 1 & 0 & $E$ & $A^{\prime}$ \\
$\beta^{\prime}$ & 1 & 0 & 1 & 1 & 0 & $F$ & $B^{\prime}$ \\
$\gamma^{\prime}$ & 1 & 1 & 0 & 1 & 0 & $G$ & $C^{\prime}$ \\
$\alpha^{\star}$ & 2 & 1 & 1 & 1 & 1 & $A^{\star}$ & $A^{\star}$ \\
$\beta^{\star}$ & 1 & 2 & 1 & 1 & 1 & $B^{\star}$ & $B^{\star}$ \\
$\gamma^{\star}$ & 1 & 1 & 2 & 1 & 1 & $C^{\star}$ & $C^{\star}$ \\
\hline
\end{tabular}

TABLE 2. Intersection numbers 
6.2.2. Case of $\Delta_{2}$. The map $\phi_{2}$ does not extend to any point of the boundary of $\Delta$, because of the collar lemma. On the contrary, $\psi_{2}$ extends continuously to the whole Thurston boundary of Teich $\left(\Sigma_{3}^{-}\right)$, for no point in the Thurston boundary satisfies $\left(\mathrm{i}_{\alpha}, \mathrm{i}_{\beta}, \mathrm{i}_{\gamma}, \mathrm{i}_{\alpha^{\prime}+\beta^{\prime}+\gamma^{\prime}}\right)=0$. The images of the vertices of the triangulation of $\operatorname{PMF}\left(\Sigma_{3}^{-}\right)$are sent to points in $\Delta_{2}$ as shown in figure 5. So the extension of $\psi_{2}$ induces a piecewise integral projective isomorphism between the Thurston boundary of Teich $\left(\Sigma_{3}^{-}\right)$and the boundary of $\Delta_{2}$.

Interpretation. The polyhedron $\Delta_{2}$ is a convex polyhedral realization of the Thurston compactification of Teich $\left(\Sigma_{3}^{-}\right)$. The map $\left(\ell_{\alpha}, \ell_{\beta}, \ell_{\gamma}, \ell_{\alpha^{\prime}+\beta^{\prime}+\gamma^{\prime}}\right)$ is an embedding of $\operatorname{Teich}\left(\Sigma_{3}^{-}\right)$onto the interior of $\Delta_{2}$. It has a continuous extension which induces a piecewise integral projective isomorphism given by $\left(\mathrm{i}_{\alpha}, \mathrm{i}_{\beta}, \mathrm{i}_{\gamma}, \mathrm{i}_{\alpha^{\prime}+\beta^{\prime}+\gamma^{\prime}}\right)$ between the Thurston boundary of Teich $\left(\Sigma_{3}^{-}\right)$and the boundary of $\Delta_{2}$.

6.3. Few words about the action of the modular group. Let $\Sigma$ be a compact surface of negative Euler-Poincaré characteristic. The mapping class group $\mathrm{MCG}(\Sigma)$ is the group of isotopy classes of diffeomorphisms of $\Sigma$ which stabilize boundary components. We call modular group, and denote $\operatorname{Mod}(\Sigma)$, the quotient of the mapping class group by its subgroup acting trivially on the Teichmüller space Teich $(\Sigma)$. It is well known that

$$
\operatorname{MCG}\left(\Sigma_{1,1}\right) \simeq \mathrm{GL}(2, \mathbf{Z}) \text { and } \operatorname{Mod}\left(\Sigma_{1,1}\right) \simeq \operatorname{PGL}(2, \mathbf{Z}),
$$

and that (direct consequence of proposition 3.1)

$$
\operatorname{MCG}\left(\Sigma_{3}^{-}\right) \simeq \mathrm{GL}(2, \mathbf{Z}) \text { and } \operatorname{Mod}\left(\Sigma_{3}^{-}\right) \simeq \operatorname{PGL}(2, \mathbf{Z}) .
$$

The modular group $\operatorname{Mod}\left(\Sigma_{3}^{-}\right)$decomposes into an amalgamated product $\mathrm{D}_{4} * \mathbf{z} / 2 \mathbf{Z} \mathrm{D}_{6}$, where each factor corresponds to some stabilisator for its action on $\operatorname{Teich}\left(\Sigma_{3}^{-}\right)$.

The modular group acts also on the boundary of the Teichmüller space, and so $\operatorname{Mod}\left(\Sigma_{3}^{-}\right)$acts on the boundary of the projective simplex $\Delta_{2}$. Clearly, every element fixes the vertex associated to $\sigma$ and stabilizes the subcomplex identified with $\partial \operatorname{Teich}\left(\Sigma_{1,1}\right)$. The elements of $\operatorname{Mod}\left(\Sigma_{3}^{-}\right)$acting projectively stabilize each set of curves $\{\alpha, \beta, \gamma\}$ and $\left\{\alpha^{\prime}, \beta^{\prime}, \gamma^{\prime}\right\}$. But, the images of $\alpha, \beta$ and $\gamma$ determine completely an element of $\operatorname{Mod}\left(\Sigma_{3}^{-}\right)$. In conclusion, the subgroup of elemets acting projectively identifies with the subroup of projective transformations of $\mathbf{P}\left(\mathbf{R}^{4}\right)$ which fix the vertex associated to $\sigma$ and permute the vertices associated to $\alpha^{\prime}, \beta^{\prime}$ and $\gamma^{\prime}$, it corresponds to the factor $\mathrm{D}_{6}$.

\section{REFERENCES}

[FLP91] Travaux de Thurston sur les surfaces, volume 66-67 of Astérisque. Société Mathématique de France, 1991.

[Gen] M. Gendulphe. Paysage systolique des surfaces hyperboliques compactes de caractéristique -1. available at http://matthieu.gendulphe.com.

[Ham03] U. Hamenstädt. Parametrizations of Teichmüller space and its Thurston boundary. In Geometric analysis and nonlinear partial differential equations, pages 81-88. Springer, 2003.

[LS04] F. Luo and R. Stong. Dehn-Thurston coordinates for curves on surfaces. Comm. Anal. Geom., 12(1-2):1-41, 2004.

[Sch82] M. Scharlemann. The complex of curves on nonorientable surfaces. J. London Math. Soc. (2), 25(1):171-184, 1982.

[Sch92] P. Schmutz. Une paramétrisation de l'espace de Teichmüller de genre $g$ donnée par $6 g-5$ géodésiques explicites. In Séminaire de Théorie Spectrale et Géométrie, No. 10, Année 1991-1992, volume 10, pages 59-64. Univ. Grenoble I, 1992. 
[Sch93] P. Schmutz. Die Parametrisierung des Teichmüllerraumes durch geodätische Längenfunktionen. Comment. Math. Helv., 68(2):278-288, 1993.

[Thu88] W. P. Thurston. On the geometry and dynamics of diffeomorphisms of surfaces. Bull. Amer. Math. Soc., 19(2):417-431, 1988.

Matthieu Gendulphe, Départment de Mathématiques, université de Fribourg, Chemin Du Musée 23, 1700 Fribourg, Suisse

E-mail address: Matthieu.Gendulphe@unifr.ch

Yohei Komori, Department of Mathematics, Osaka City University, 558-8585, Osaka, JAPAN

E-mail address: komori@sci.osaka-cu.ac.jp 